\title{
Death by Opioids: Are there non-addictive scientific solutions?
}

\author{
B William Downs ${ }^{1}$, Kenneth Blum ${ }^{1-6 *}$, David Baron ${ }^{2}$, Abdalla Bowirrat $^{7}$, Lisa Lott $^{6}$, Raymond Brewer ${ }^{6}$, Brent Boyett ${ }^{3}$, David Siwicki ${ }^{6}$, A Kenison Roy \\ $111^{8}$, Arwen Podesta ${ }^{8}$, Sampada Badgaiyan ${ }^{6}$, Raju Hajela $^{9}$, Lyle Fried ${ }^{10}$ and Rajendra D Badgaiyan $^{11}$ \\ ${ }^{1}$ Department of Nutrigenomic Research, Victory Nutrition International, Inc., Lederach, PA, USA \\ ${ }^{2}$ Western University, Health Sciences, Graduate School of Biomedical Sciences, Pomona, CA, USA \\ ${ }^{3}$ Division of Neuroscience and Addiction Research, Pathway Healthcare, Birmingham, AL, USA \\ ${ }^{4}$ Eotvos Loránd University, Institute of Psychology, Budapest, Hungary \\ ${ }^{5}$ Department of Psychiatry, Wright State University Boonshoft School of Medicine and Dayton VA Medical Center, Dayton, OH, USA \\ ${ }^{6}$ Division of Precision Nutrigenomics, Geneus Health, LLC., San Antonio, TX, USA \\ ${ }^{7}$ Department of Neuroscience and Genetics, Interdisciplinary Center Herzliya, Israel \\ ${ }^{8}$ Department of Psychiatry, Tulane University School of Medicine, New Orleans, LA, USA \\ ${ }^{9}$ Department of Family Medicine, Cumming School of Medicine, University of Calgary, AB, Canada \\ ${ }^{10}$ Transformations Treatment Center, Delray Beach, FL, USA \\ ${ }^{11}$ Department of Psychiatry, South Texas Veteran Health Care System, Audie L. Murphy Memorial VA Hospital, San Antonio, TX, Long School of Medicine, \\ University of Texas Medical Center, San Antonio, TX, USA
}

\begin{abstract}
In the face of the current Opioid crisis in America killing close to 800,000 people since 2004, we are proposing a novel approach to assist in at least attenuating these unwanted premature deaths. While we applaud the wonderful efforts of our governmental institutes and professional societies (NIDA, NIAAA, ASAM, ABAM ) in their extraordinary efforts in combating this continued dilemma, the current approach is failing, and other alternative approaches should at least be tested. These truths present a serious ethical dilemma to scientists, clinicians and counselors in the Reward Deficiency Syndrome (RDS) treatment community. It is important to realize that the current DSM-5 does not actually accurately display the natural brain reward process. The human brain has not been designed to carve out specific drugs like opioids, alcohol, nicotine, cocaine, benzodiazepines or cannabis and process addictions such as gambling as distinct endophenotypes. This is true in spite of natural ligands for cannabinoids, endorphins, or even benzodiazepines. The most accurate endophenotype is indeed reward dysfunction (e.g hypodopaminergic or hyperdopaminergic). With this mind, we are hereby proposing that the current Medication Assisted Treatment (i.e. 'MAT') expands to needed individuals as an initial "Band-Aid" to reduce harm avoidance, with the long-term goal of prophylaxis. So, to be clear, there may be other promising modalities other than MAT such as repetitive transcranial magnetic stimulation (rTMS), exercise and even new medications with positive allosteric modulators of GABA-A receptors, as well as the highly researched Genetic Addiction Risk Score (GARS) coupled with precision KB220Z. This will induce "dopamine homeostasis" to effectively rebalance and restore healthier brain function by promoting the cross talk between various brain regions (e.g. Nucleus accumbens, cingulate gyrus, hippocampus etc.) resulting in dopamine homeostasis. Our laudable goal is to not only save lives, but to redeem joy and improve the quality of life in the recovery community through scientifically sound natural non-addicting alternatives.
\end{abstract}

Since 2000, the turn of the century, the total number of prescription analgesic opioids sold in America has almost quadrupled. Moreover, while unimaginable during the same period, prescription opioid fatalities have more than quadrupled. It is notable, for example, West Virginia has the highest overdose death rate in the nation-35 per every 100,000 people. Along these lines it is not surprising that West Virginia is the site of dozens of lawsuits filed against not only drug wholesalers, but physicians and even pharmacists who may have been directly or indirectly involved in and/or contributed to opioid distribution. In essence, these and other lawsuits claim that physicians, pharmaceutical companies, and "pill mills" exploited patients, got them hooked on pills, and cost individuals and the state millions of dollars [1-2]. When you add up all the states in America, the total economic cost is north of one trillion dollars.

A recent analysis from Stanford University suggested that without any changes in currently available treatment, prevention, and public health approaches, we should expect to have 510,000 deaths from prescription opioids and street heroin from 2016 to 2025 in the US [3]. With this mind, our purpose in writing this editorial is not to reprimand anyone, but to point out the importance of a change in focus as it relates to out-of-the-box thinking based on scientific fact. This refocusing may not be at first financially beneficial to the owners of Suboxone clinics here in the USA. We sincerely believe that most physicians (especially owners) want to do the right thing. But, the structure, treatment dogma

${ }^{\star}$ Correspondence to: Blum K, Western University Health Sciences Graduate School of Biomedical Sciences, Pomona, CA, USA, E-mail: Drd2gene@gmail.com

Key words: american opioid crisis, hyperdopaminergia, hypodopaminergia, dopamine homeostasis, genetic addiction risk score(gars), pro-dopamine regulation

Received: October 14, 2019; Accepted: October 28, 2019; Published: October 31, 2019 
and objectives of the current system add tempting financial barriers $\mathrm{d}$ by a financial barrier to continue with the known and unfortunate "revolving door."

We are compelled to provide some rationale related to why it is important to more carefully evaluate the current failed approach to help addicted patients on opioids have a better outcome and subsequently an improved quality of life in recovery, a laudable goal.

As scientists and clinicians, we need to face the truth of two important citations by Albert Einstein.

1. "Insanity is doing the same thing over and over and expecting different results."

2. "Given one hour to save the world, I would spend 55 minutes defining the problem and 5 minutes finding the solution."

Stating \#2 differently, "If you want to find a solution, you must first be able to accurately state or define the problem." These truths present a serious ethical dilemma to scientists, clinicians and counselors in the Reward Deficiency Syndrome (RDS) treatment community [4]. It is important to realize that the current DSM-5 does not actually accurately display the natural brain reward process. The human brain has not been designed to carve out specific drugs like opioids, alcohol, nicotine, cocaine, benzodiazepines or cannabis and process addictions such as gambling, as distinct endophenotypes. This is true in spite of natural ligands for cannabinoids, endorphins, or even benzodiazepines. The most accurate endophenotype is indeed reward dysfunction (e. g hypodopaminergic or hyperdopaminergic). Approved and popular treatment protocols 'follow the painted footsteps' of conventional 'Standard of Care' compliant programs, which have, for the most part, not achieved sustainable long-term abstinence. These programs are intended to get the addict 'clean' (i.e. detoxified) during his/her time in a rehab facility or an outpatient program. Once the individual is considered 'clean', he/she is released for reintroduction back into society. Recovering addicts may attend different types of fellowship programs, such as AA, Celebrate Recovery, etc. [5]. But the published statistics that demonstrate a high rate of relapse indicate that we are not solving the problem; in fact it is growing - the epidemic continues [6]. Moreover, most rehabilitation programs use some sort of opioid therapy to treat an opioid addiction. It is our opinion, that albeit harm reduction, even that statement sounds absurd for solving the opioid addiction crisis [6].

The use of legally prescribed opioids and illegal drugs to 'feel better' has unquestionably reached epidemic status. What's even more alarming is the rate of relapse and excessive re-indulgence in abusable drugs and alcohol. As a community of scientists and clinicians, it is incumbent upon us to implement different strategies that will change the course of treatment objectives and outcomes. While we applaud the enormous efforts of the National Institute of Drug Abuse (NIDA) and the National Institute of Alcohol Abuse and Alcoholism (NIAAA) in their fight to attenuate or resolve our current drug epidemic, out of box thinking based on scientific evidence must reign.

Most of the scientific community is aware of the story of Dr. Ignaz Semmelweis. The following section and citations are excerpted from an article published in RPubs [7]. "In 1847 the Hungarian physician Ignaz Semmelweis noted that childbed fever was a deadly disease affecting women that had just given birth. Semmelweis was puzzled about this terrible occurrence because in the early 1840s at the Vienna General Hospital as many as $10 \%$ of the women giving birth died from it." Semmelweis did research on women giving birth at two clinics at the Vienna General Hospital for the years 1841 to 1846 . He noted a significant difference in the incidence of puerperal fever deaths, also known as childbed fever, between the two clinics. When Semmelweis crunched the numbers, he discovered that women in clinic 1, staffed by doctors and medical students, died at a rate nearly five times higher than women in clinic \#2, which was staffed by midwives. Interestingly, midwives worked only with the women giving birth; the medical students and doctors also spent time in the autopsy rooms examining corpses.

After close scrutiny and extensive observations, Semmelweis concluded that 'something' that spread from the hands of medical students and doctors who had worked on the corpses is what actually caused the childbed fever. In a desperate attempt to stop the high mortality rates, he insisted that they wash their hands! This was an unorthodox and controversial request at the time as none of the doctors in Vienna knew about bacteria back then.

Despite the compelling evidence, Semmelweis' theory that childbed fever was caused by some "substance" (we know was bacteria) from autopsy room corpses was ridiculed by contemporary physicians and scientists. You'd think everyone would have been thrilled that Semmelweis had solved the problem! But they weren't thrilled. Doctors were upset because Semmelweis' hypothesis made it look like they were responsible for giving childbed fever to the women. The medical community largely rejected his discovery and in 1849 he lost his job and left the Vienna General Hospital to get away from the rejection and harsh criticisms levied against him by colleagues. In 1865, when he was only 47 years old, Ignaz Semmelweis was committed to a mental asylum.

Semmelweis did provide, through diligent scientific observation, compelling data to inform the doctors he attributed the cause of childbed fever to the only possible cause - contaminated hands of the doctors and students. They rejected the evidence-based revelation; many more women and babies died as a result. This is a sad indictment against his medical peers and the stubborn mindset to remain entrenched in conventional dogma.

By default, an invention is outside the box of conventional thought. An invention should make such an innovative contribution that it tips an entire system into a new trajectory. However, from a market acceptance perspective, new ideas and inventions go through 3 phases in the market cycle:

\section{Outright rejection}

\section{Analysis, suspicion and criticism}

\section{Acceptance and accolades}

Some inventions that went through these stages were the light bulb, the bicycle, the automobile, liquid fingernail polish, the cheeseburger, talking motion pictures, telephone answering machines, and even the laptop computer.

There is another factor that slows acceptance of new technologies; the vested financial interests in the conventional way of doing things. The incentive to reduce drug usage; excessive unhealthy, and even dangerous RDS behaviors; and improve immediate and sustainable recovery rates may be opposed to revenue incentives driving pharmaceutical companies. There are approximately 14,500 clinics and programs in America that provide treatment for all types of addictive behaviors that are termed "RDS". We are proposing that these important treatment providers consider novel ways to improve outcomes and 


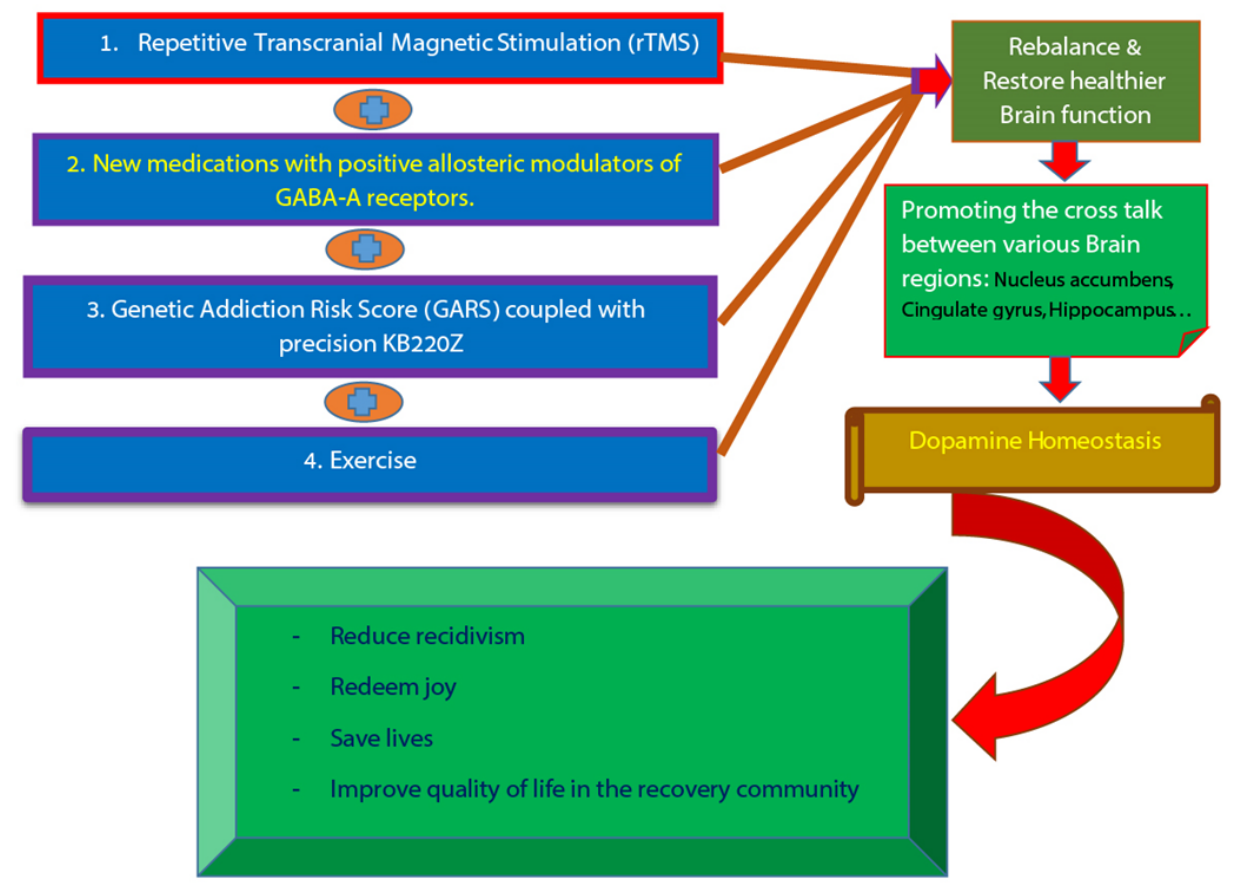

Figure 1. Novel approach to assist or attenuating unwanted premature deaths as a result of Opioid crisis-other than Medication Assisted Treatment (MAT) - through scientifically sound natural non-addicting alternatives

even compliance to MAT. While we applaud their continued efforts to reduce unwanted opioid use disorder and even alcohol and other drugs [8] we suggest a reconsideration of best practice. Our basic question is should we continue to provide opioids for opioid recovery in the long term. While we agree that buprenorphine combinations reduce societal harm and naltrexone injectable maybe a real important clinical option, can we find ways to promote 'dopamine homeostasis" [9]. We are cognizant that most clinics want to do the right thing and strive to reduce recidivism rates [10].

Finally, the epidemic of not only opioid use, but prescription and illicit drug use has escalated the rates of RDS behaviors that endanger societal health. Physicians and other healthcare professionals need to reexamine the routine practice of prescribing opioids for pain and recovery. Continuing in this path will not produce different results. To be clear there may be other promising modalities other than MAT such as repetitive transcranial magnetic stimulation (rTMS) [10], exercise [11] and even new medications with positive allosteric modulators of GABA-A receptors [12], as well as the highly researched Genetic Addiction Risk Score (GARS) coupled with precision KB220Z to induce dopamine homeostasis [13] to effectively rebalance and restore healthier brain function by promoting effective cross talk between various brain regions (e.g. Nucleus accumbens, cingulate gyrus, hippocampus etc.) resulting in dopamine homeostasis $[14,15]$ The ASAM definition of Addiction, which encompasses bio-psycho-social-genetic-spiritual factors, punctuates the need to provide comprehensive treatments and guide affected individuals to holistic recovery (Figure 1).

New exciting modalities, as they become available following required validating research, certainly need to be implemented in all treatment programs in America. Anything less will ultimately protect the so called "revolving door" treatment paradigm for as many as $90 \%$ of returning treatment participants. It is time to adhere to genuine scientific principles.

\section{Acknowledgement}

We appreciate the expert edits of Margaret A. Madigan, RN.

\section{Author contribution}

$\mathrm{KB}$ and $\mathrm{BWD}$ wrote the original manuscript followed by all coauthors commenting on the original manuscript and approved the final work.

\section{Conflicts of interest}

Kenneth Blum, $\mathrm{PhD}$ through his companies and patents related to Pro-dopamine regulation (Kb220 and variants) have been worldwide exclusively licensed to Geneus Health, LLC. Moreover, genetic patents related to GARS are owned by Geneus Health, LLC. Kenneth Blum and David Siwicki through their company IGENE, LLC have percent ownership in Geneus Health. B.W. Downs is founder and CEO of Victory Nutrition International, a licensee of Geneus Health. Dr. Blum is on their Scientific Advisory Board. Drs. Lisa Lott and Raymond Brewer are paid consultants of Geneus Health. Drs. Blum (Chair), Baron, Siwicki, Brewer, Roy, and Badgaiyan are members of Geneus Health, Scientific Board. Dr. Blum is paid consultant of Pathway Healthcare. There are no other conflicts of interest.

\section{Funding}

Kenneth Blum, $\mathrm{PhD}$ is the recipient a NIH grant along with Marjorie C. Gondre-Lewis of Howard University, grant \# R41 MD012318/MD/ NIMHD NIH HHS/United States.

\section{References}

1. West JC (2014) Case law update. United States v Gonzales-Ramos, Case No. 2:13 cr-00105 (SD W Va September 3, 2013). J Healthc Risk Manag 33: 48-49. [Crossref]

2. Blum K, Gold M, Clark HW, Dushaj K, Badgaiyan RD, et al. (2016) Should the United States government repeal restrictions on buprenorphine/naloxone treatment? Subst Use Misuse 51: 1674-1679. 
3. Srivastava AB, Gold MS (2018) Beyond supply: How see must tackle the opioid epidemic. Mayo Clin Proc 93: 269-272. [Crossref]

4. Blum K, Chen ALC, Thanos PK (2018) Genetic addiction risk score (GARS), a predictor of vulnerability to opioid dependence. Front Biosci (Elite Ed) 10: 175-196.

5. Blum K, Thompson B, Demotrovics Z, et al. (2015) The molecular neurobiology of twelve steps program \& fellowship: connecting the dots for recovery. J Reward Defic Syndr 1: 46-64.

6. Pitt AL, Humphreys K, Brandeau ML (2018) Modeling health benefits and harms of public policy responses to the US opioid epidemic. Am J Public Health 108: 13941400 .

7. Davis R (2015) The doctor who championed handwashing and briefly saved lives. National Public Radio.

8. The Lancet Psychiatry (2017) Money, money, money. Lancet Psychiatry 4: 649. [Crossref]

9. Miller D, Miller M, Blum K, Badgaiyan RD, Febo M (2015) Addiction treatment in america: after money or aftercare? J Reward Defic Syndr 1: 87-94. [Crossref]
10. Makani R, Pradhan B, Shah U, Parikh T (2017) Role of repetitive transcranial magnetic stimulation (rtms) in treatment of addiction and related disorders: A systematic review. Curr Drug Abuse Rev 10: 31-43.

11. Robison LS, Swenson S, Hamilton J, Thanos PK (2018) Exercise reduces dopamine D1R and increases D2R in rats: implications for addiction. Med Sci Sports Exerc 50: 1596-1602. [Crossref]

12. Pin JP, Prezeau L (2015) Allosteric modulators of GABA(B) receptors: mechanism of action and therapeutic perspective. Curr Neuropharmacol 5: 195-201.

13. Blum K, Oscar-Berman M, Demetrovics Z, Barh D, Gold MS, et al. (2014) Genetic addiction risk score (GARS): molecular neurogenetic evidence for predisposition to reward deficiency syndrome (RDS). Mol Neurobiol 50: 765-796.

14. Miller M, Chen AL, Stokes SD (2012) Early intervention of intravenous KB220IV-neuroadaptagen amino-acid therapy (NAAT) improves behavioural outcomes in a residential addiction treatment program: a pilot study. J Psychoactive Drugs 44: 398409 .

15. Blum K, Liu Y, Wang W (2015) rsfMRI effects of KB220Z on neural pathways in reward circuitry of abstinent genotyped heroin addicts. Postgrad Med 127: 232-241.

Copyright: $₫ 2019$ Downs BW. This is an open-access article distributed under the terms of the Creative Commons Attribution License, which permits unrestricted use, distribution, and reproduction in any medium, provided the original author and source are credited. 